\title{
Bilateral Risk-Reducing Mastectomies with Implant-Based Reconstructions Followed Long Term: A Consecutive Series of 185 Patients
}

\author{
Karin Isaksson ${ }^{1}$ Brita Arver ${ }^{2} \cdot$ Matteo Bottai $^{3} \cdot$ Andreas Pettersson $^{4} \cdot$ Marie Wickman $^{1,5} \cdot$ \\ Kerstin Sandelin ${ }^{1}$
}

\begin{abstract}
Background Bilateral risk-reducing mastectomy (BRRM) is the most effective method to prevent breast cancer in genetically predisposed women and is often performed concomitantly with breast reconstruction. The reconstruction time varies and corrective surgeries are common.

Methods This study evaluated 185 consecutive cases of BRRM and immediate breast reconstruction with implants with regard to surgical outcome and resource consumption. With an 18-year observation period, it was possible to compare permanent expander implants (PEIs) with permanent fixed volume implants (PIs).

Results A minimum follow-up of 2 years for all participants but one was achieved. Seventy-five percent $(n=138)$ of the women received PEI and $25 \%(n=47)$ PI. In a multivariate analysis including age, BMI $(<25, \geq 25)$, smoking (yes, no), implant type (PEI, PI), incision technique, operation time and specimen weight $<350 \mathrm{~g}, \geq 350 \mathrm{~g}$ ), only $\mathrm{BMI} \geq 25$ was associated with an increased risk of an early complication (OR 7.1, 95\% CI 2.44-20.4). As expected, there was a significant difference in median reconstruction time between PEI and PI (12.4 vs. 1.0 months, $p<0.001)$. The cumulative reoperation-free 2-year survival was significantly higher in the PI than in the PEI group $(81 \%$ vs. $26 \%, p<0.001)$.

Conclusion Implant-based reconstruction remains a valid option after BRRM in high-risk women. Whenever possible (low BMI and small breast volume without severe ptosis), permanent fixed volume implants can be safely recommended and are resource saving in comparison with permanent expander implants.
\end{abstract}

Kerstin Sandelin

kerstin.sandelin@ki.se

1 Department of Molecular Medicine and Surgery, Karolinska Institutet, Stockholm, Sweden

2 Department of Oncology and Pathology, Karolinska Institutet, Stockholm, Sweden

3 Division of Biostatistics, Institute of Environmental Medicine, Karolinska Institutet, Stockholm, Sweden

4 Clinical Epidemiology Unit, Department of Medicine, Karolinska Institutet, Solna, Stockholm, Sweden

5 Sophiahemmet University, Stockholm, Sweden

\section{Introduction}

Bilateral risk-reducing mastectomy (BRRM) is the most effective method to prevent breast cancer in genetically predisposed women. The relative risk reduction in BRCA1 and BRCA2 mutation carriers is $>90 \%$ [1-3]. Prior studies have shown that BRRM reduces psychological distress but has a negative impact on sexuality and body image $[4,5]$. Most women are premenopausal when undergoing BRRM and opt for immediate breast reconstruction (IBR) with either implants or autologous tissue [6]. Even though cancer prevention is the primary goal, cosmesis is also of great importance. Satisfactory cosmetic outcome of breast reconstruction generally requires more than one operation. 
A Swedish nationwide survey of 223 women undergoing BRRM, 220 of whom also underwent breast reconstruction, concluded that the procedure is safe and efficacious in reducing future breast cancer risk. Complications and reoperations associated with the reconstructive procedure were, however, common [7]. Implant-based reconstruction was the most frequently used $(95 \%)$ reconstruction method.

The Karolinska University Hospital serves as a referral center for women at hereditary increased risk of breast cancer in the Stockholm County Council (catchment area population of 2.2 million) and performs about $50 \%$ of all BRRMs in Sweden. A series of publications from the Karolinska have focused on the short-term procedural aspects of BRRM and on the patients' experiences [4, 7-11]. There are few long-term follow-up studies addressing complications and reoperations after BRRM with IBR in women without breast cancer.

The surgical development in BRRM has gone from nonskin-sparing mastectomies to mastectomies where the skin and-if suitable - the nipple-areola complex (NAC) are preserved, so-called conservative mastectomies [12-14]. With a larger skin envelope left intact, the tissue expander implants could in many cases be replaced by permanent fixed volume implants. The primary aim of this study was to retrospectively assess complications, reoperations and healthcare utilization among a consecutive series of 185 women undergoing BRRM and IBR with implants. A secondary aim was to compare the expander and permanent fixed volume implant reconstructions according to the above parameters.

\section{Materials and methods}

\section{Patients}

This retrospective follow-up study included a series of 185 consecutive women at increased risk of breast cancer who underwent BRRM at the Karolinska University Hospital, Sweden, between 1993 and 2010. All women were offered and accepted IBR, which was performed with either permanent silicone/saline expander implants with detachable filling ports (PEI) or permanent silicone implants with a fixed volume (PI). All implants were placed subpectoral with total muscular coverage. A subset of 103 participants, operated on between 1995 and 2005, were also part of the prior national survey addressing the efficacy of BRRM and regional disparities [7]. Women with a history of breast cancer were excluded. One patient reconstructed with temporary saline expanders followed by permanent implants and two patients reconstructed with autologous tissue were also excluded. The study was approved by the Regional Ethics Committee in Stockholm.

\section{Surgical protocol and follow-up}

The routines regarding surveillance, preoperative investigation and surgical care have been described in detail in previous publications [4, 7, 10, 15]. The BRRMs and IBRs were carried out jointly by a breast surgeon and a reconstructive plastic surgeon. In this study, $80 \%$ of the mastectomies were performed by three breast surgeons and $95 \%$ of the reconstructions by three plastic surgeons.

During the 1990s, increasingly skin-sparing mastectomies were performed. The NAC was excised through an oval, horizontal skin incision, leaving two skin flaps. If the nipple base biopsy was benign, the nipple tip was regrafted. Since 2002, NAC-sparing procedures have dominated. For these patients, a supra-areolar, omega-type incision, sometimes with removal of a skin oval to achieve a NAClift, has been used. In large or pendulous breasts, a modified Wise pattern incision with nipple regrafting is chosen (Figs. 1, 2, 3).

In the beginning of the study, permanent expander implants, either round or anatomically shaped, with detachable filling ports used for saline filling under the expansion period, dominated. Since the mid-2000s, anatomically shaped, textured, permanent silicone implants have replaced most expander implants for small to moderate breast volumes, i.e., an estimated breast volume of less than $350 \mathrm{cc}$.

All patients received intravenous antibiotic prophylaxis, either a single dose of $2 \mathrm{~g}$ cloxacillin or $600 \mathrm{mg}$ clindamycin. Two drains were used for each breast, one in the implant pocket and one in the subcutaneous space. For expander-based reconstructions, expansions were performed in the outpatient clinic by a trained nurse, usually for a time period of 23 months. After completed size adjustments, including a period of overexpansion, the filling ports were removed. If the areolae had been excised, tattooing was most often performed. During the study period, all women remained in regular contact with the hereditary high-risk clinic after completion of reconstruction. The reconstructive surgical team saw patients when needed.

\section{Data collection}

The data collection sheet was an extended version of the sheet used in the national survey [7]. Computerized hospital records enabled access to detailed clinical information throughout the reconstruction process. Information was extracted on age, mutation status, medical history, smoking habits, body mass index (BMI), mastectomy and reconstructive techniques, specimen weights, histopathology and early postoperative complications ( $<30$ days). Early complications included surgical site infection (SSI) (defined as 
Fig. 1 a Preoperative drawing for oval incisions.

b Reconstruction complete with permanent expander implants, regrafted nipples and tattooing of the areolae

Fig. 2 a Preoperative picture of a patient with estimated breast volume $175 \mathrm{cc}$.

b Reconstruction complete with periareolar incisions and permanent implants

Fig. 3 a Preoperative picture of a patient with ptotic breasts.

b Reconstruction complete with modified Wise pattern incisions, regrafted nipples and tattooing of the areolae
A

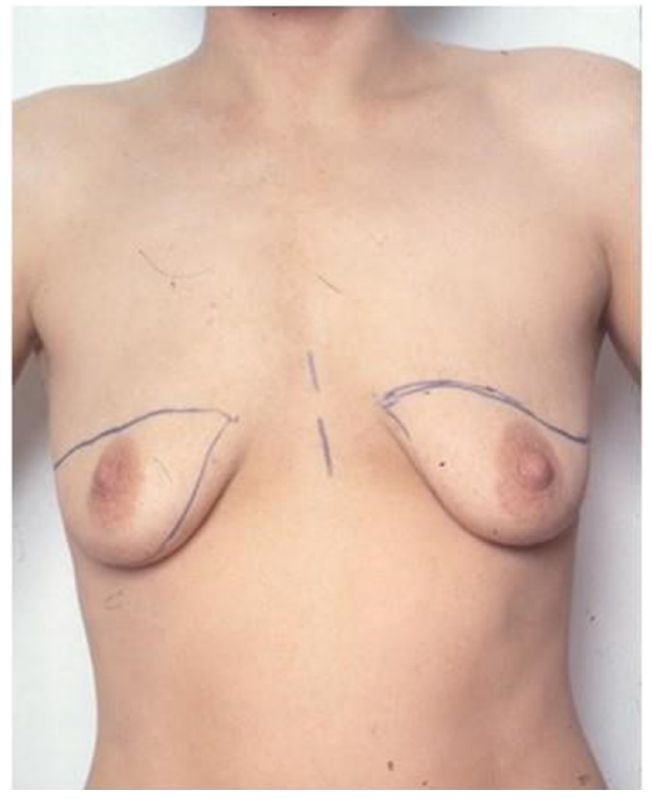

B

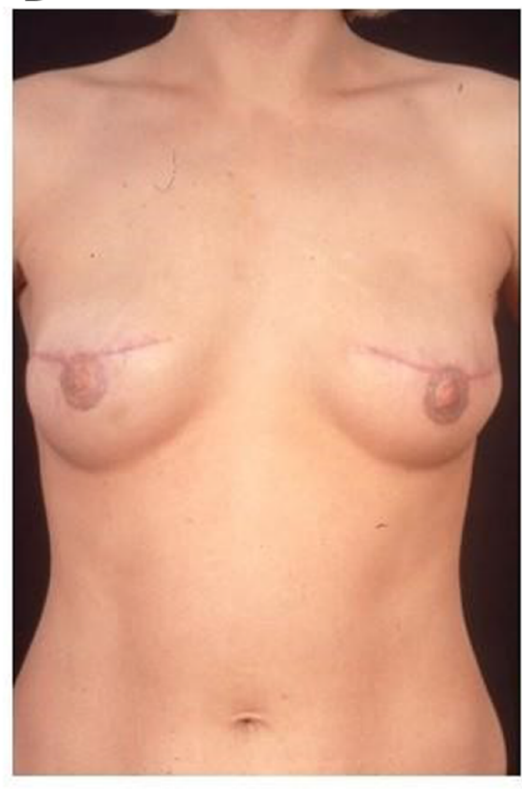

A
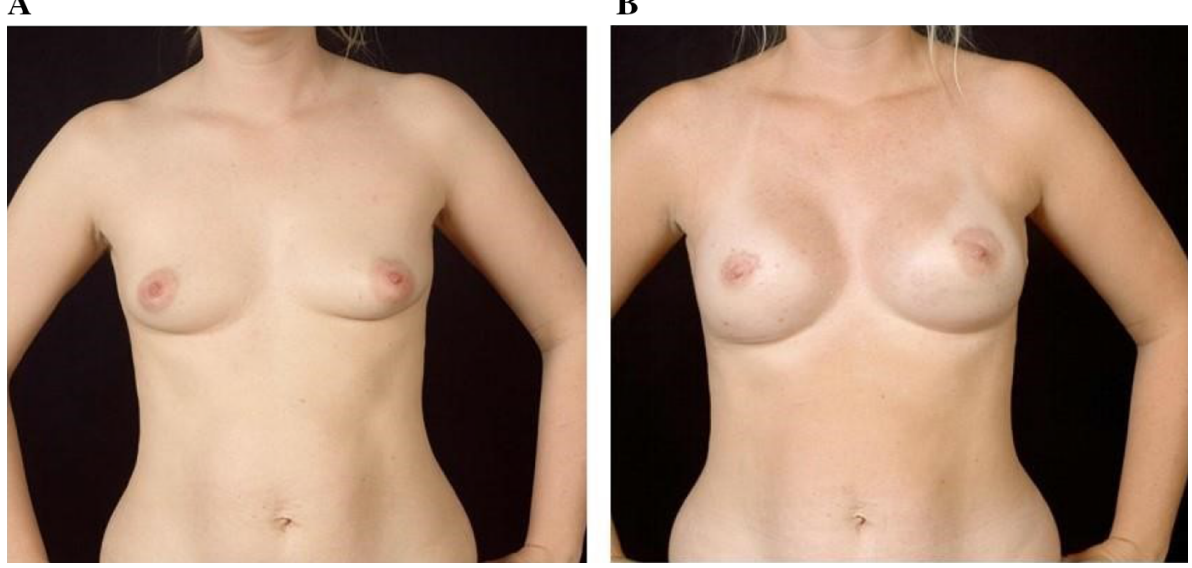

A

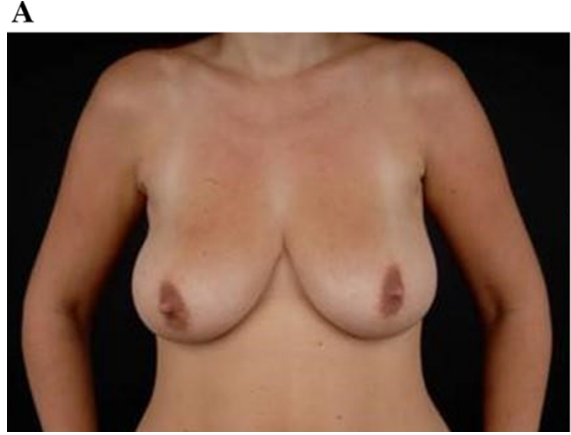

B

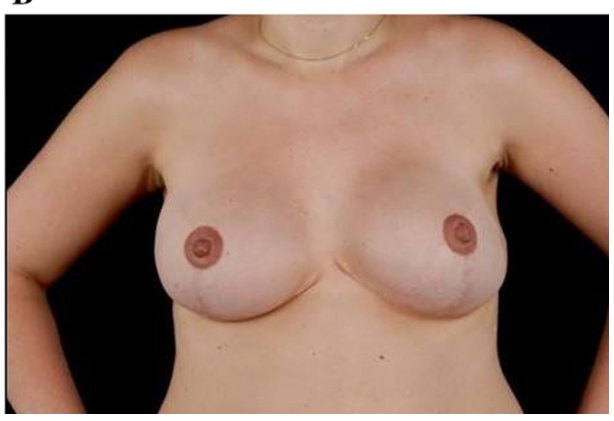


a clinical suspicion of infection, with or without positive microbiological culture, leading to antibiotic treatment), hematoma requiring reoperation, blood loss requiring red blood cell transfusion, seroma requiring aspiration and wound dehiscence. Non-breast-related events (e.g., pneumothorax) were recorded. Data on unanticipated corrective procedures, number of postoperative outpatient visits and cancer diagnoses post-BRRM were also collected.

In women with PEI, the reconstruction period was considered complete when all expansions had been conducted, the filling ports removed (if desired by the patient), the areolae tattooed (performed in the vast majority of cases) and no further corrective procedures were planned. For women with PI with NAC preservation, the reconstruction was considered complete after a month of healing. An unanticipated reoperation was defined as a surgical procedure not being part of the planned reconstruction, i.e., all surgeries after the BRRM with IBR except for removal of the filling port. If any such procedures were performed (or scheduled for) during the first 6 months after completion, this prolonged the reconstructive time until 30 days after the corrective surgery. Tattooing was not defined as a surgical procedure.

The medical charts were reviewed to obtain a minimum follow-up of 2 years for all participants. Follow-up for each woman continued from the date of mastectomy until the last medical chart review or death. Nineteen patients that had either emigrated from the Stockholm County or been referred to Karolinska from other regions of the country were contacted by telephone. We were unable to reach two patients, and for these, the date of the last clinical visit was defined as the end of follow-up. Vital status for all 185 patients was checked in December 2016.

\section{Statistical analysis}

Baseline patient characteristics and measures of utilization of healthcare resources were compared between PI and PEI. Numerical variables were tested with Wilcoxon's rank-sum test and categorical variables with Fisher's exact test. The probability of early postoperative complication was modeled with logistic regression. When comparing the PI and the PEI groups, crude and adjusted probabilities and odds ratios were obtained along with their respective $95 \%$ confidence intervals and p-values. Linear regression was used to model the mean of numeric outcome variables. Estimated means and mean differences were obtained with their respective $95 \%$ confidence intervals and $p$-values. The robust sandwich estimator was used to obtain estimates for the standard errors.

Outcome variables that represented time to non-repeating events (e.g., death) were analyzed with the KaplanMeier method and modeled with Cox regression. The former allowed estimating survival probability over follow-up time and the latter estimating crude and adjusted hazard ratios with their respective $95 \%$ confidence intervals and $\mathrm{p}$-values. P-values less than 0.05 were considered statistically significant. All the statistical analyses were performed with Stata version 14 (StataCorp, College Station, TX).

\section{Results}

\section{Patients}

Out of the 185 women, 101 were BRCA1/2 mutation carriers $(54.6 \%)$ (Table 1$)$. Of the 84 women with no proven mutation, all but four had an estimated $30 \%$ or higher lifetime risk of breast cancer. The risk estimation was made using Claus tables and later in the study BOADICEA (Breast and Ovarian Analysis of Disease Incidence and Carrier Estimation Algorithm) as referred to in our previous publication [7]. Five mutation carriers underwent bilateral risk-reducing salpingo-oophorectomy (BRRSO) at the same time as BRRM. Five women had previously been treated for ovarian cancer but were considered disease-free when accepted for BRRM. Apart from three women with type II diabetes, no woman in the cohort had been diagnosed with any serious medical condition.

Seventy-five percent $(n=138)$ of the women received PEI and 25\% $(n=47)$ PI. During the study period, the annual number of operations increased, and PI became more prevalent (Fig. 4). During 2007-2010, more than half of the women received PI.

The most common type of incision during the first 8 years of the study was the oval excision, where the NAC was excised and the nipple tip regrafted ( $n=40,21.6 \%$ during the whole study period) (Fig. 1a, b). This technique was then replaced by the periareolar incision with preservation of the NAC $(n=95,51.4 \%)$ (Fig. 2a, b). In seven cases $(3.8 \%)$, a transareolar incision with nipple tip regrafting was made. In large or pendulous breasts, a modified Wise pattern incision was the method of choice, where the NAC was removed and the nipple tip usually regrafted ( $n=43,23.2 \%$ ) (Fig. 3a, b).

Patient characteristics were similar in the PEI and PI groups with respect to age, mutation status and smoking habits (Table 1). Median BMI and specimen weight were significantly higher in the PEI group, and the operating time was longer.

Nineteen women $(10.3 \%)$ had abnormal findings in the breast specimens including two small $(<0 \mathrm{~mm})$ invasive ductal carcinomas.

Median follow-up time for the whole cohort was 6.1 years (range 1.1-18.5), being significantly longer for 
Table 1 Characteristics of 185 women undergoing BRRM with IBR 1993-2010

\begin{tabular}{|c|c|c|c|c|}
\hline & $\begin{array}{l}\text { All } \\
n=185\end{array}$ & $\begin{array}{l}\text { PI } \\
n=47\end{array}$ & $\begin{array}{l}\text { PEI } \\
n=138\end{array}$ & $p^{*}$ \\
\hline Median age at BRRM, years (range) & $39.6(22.8-68.9)$ & $39.7(23.0-62.1)$ & $39.6(22.8-68.9)$ & 0.877 \\
\hline BRCA1/2 mutation carrier, $n(\%)$ & $101(54.6)$ & $25(53.2)$ & $76(55.1)$ & 0.866 \\
\hline Median $\mathrm{BMI}^{\mathrm{a}}, \mathrm{kg} / \mathrm{m}^{2}$ (range) & $22.6(16.6-40.1)$ & $21.3(18.1-30.7)$ & $23.1(16.6-40.1)$ & 0.000 \\
\hline Smoking ${ }^{\mathrm{b}}, n(\%)$ & $30(18.1)$ & $4(9.1)$ & $26(21.3)$ & 0.108 \\
\hline Type II diabetes, $n(\%)$ & $3(1.6)$ & $1(2.1)$ & $2(1.5)$ & 1.000 \\
\hline Ovarian cancer before BRRM, $n(\%)$ & $5(2.7)$ & $2(4.3)$ & $3(2.2)$ & 0.602 \\
\hline Median operating time, min (range) & $153.0(75.0-270.0)$ & $129.0(75.0-203.0)$ & $159.0(90.0-270.0)$ & 0.000 \\
\hline Median specimen weight ${ }^{\mathrm{c}}, \mathrm{g}$ (range) & $312.5(45.0-1923.5)$ & $243.8(69.0-844.0)$ & $372.5(45.0-1923.5)$ & 0.000 \\
\hline Median follow-up, years (ranged) & $6.1(1.1-18.5)$ & $3.4(2.0-15.1)$ & $7.4(1.1-18.5)$ & 0.000 \\
\hline
\end{tabular}

$B R R M$ bilateral risk-reducing mastectomy, $I B R$ immediate breast reconstruction, $P I$ permanent fixed volume implant, $P E I$ permanent expander implant

*The difference between PI and PEI

${ }^{\mathrm{a}}$ Missing data for three patients $(1.6 \%)$

${ }^{\mathrm{b}}$ Missing data for 19 patients $(10.3 \%)$

${ }^{\mathrm{c}}$ Missing data for five breasts $(1.4 \%)$

${ }^{\mathrm{d}}$ All women but one had a minimum follow-up of 2 years

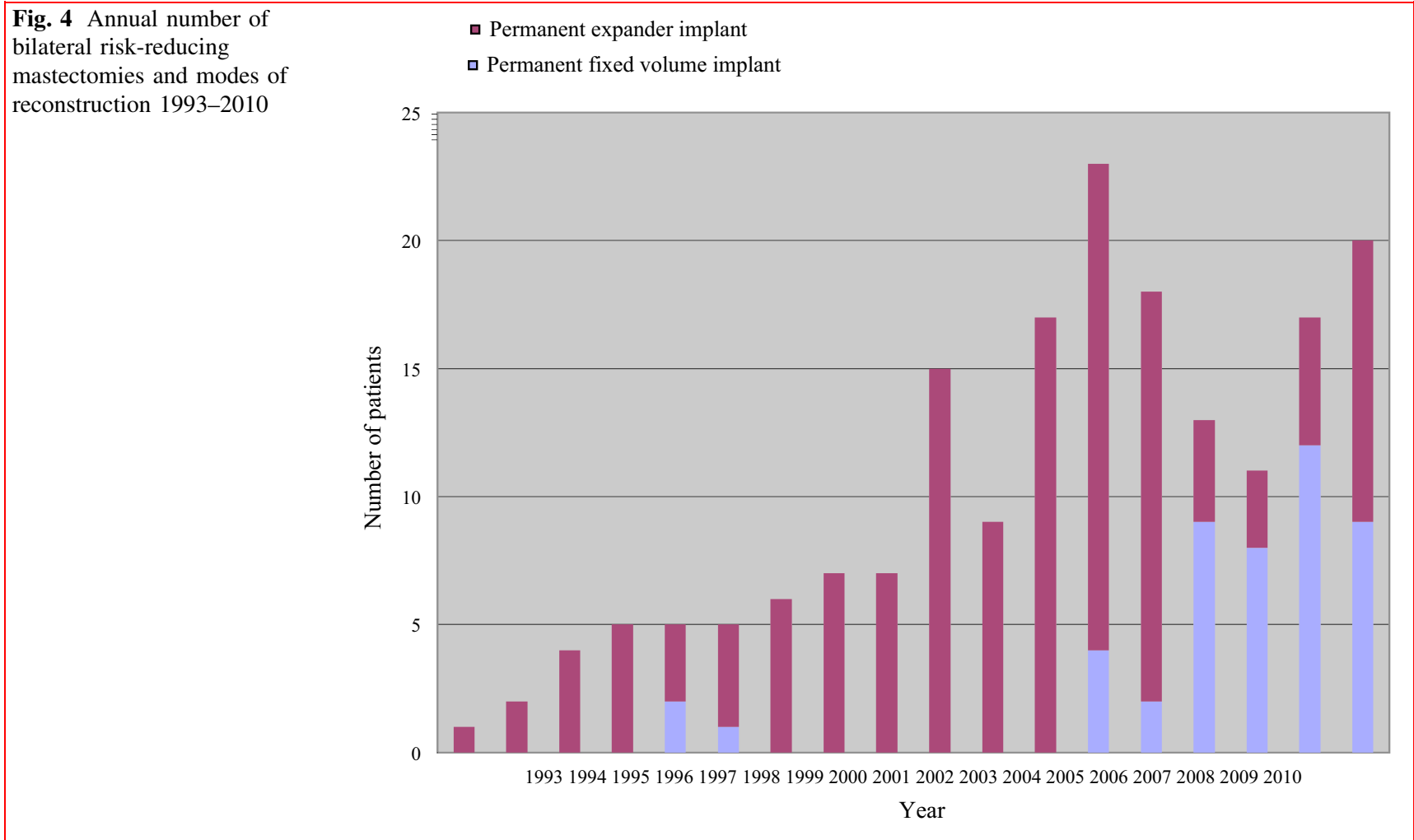

the PEI group (median 7.4 years; range $1.1-18.5$ years) than for the PI group (3.4 years; range 2.0-15.1 years). All women but one had a minimum follow-up of 2 years. The woman with shorter follow-up was from another region of the country, underwent BRRM in 2007 and was lost to follow-up 1.1 year later.

\section{Early postoperative complications (within 30 days)}

Eighty-eight women (47.6\%) experienced at least one early postoperative complication (Table 2), the most common being SSI ( $n=42,22.7 \%)$, leading to implant loss in ten cases $(5.4 \%)$. Necrosis of the wound edges or the nipple 
Table 2 Early postoperative complications ( $<30$ days) in 185 women undergoing BRRM with IBR

\begin{tabular}{|c|c|c|c|c|}
\hline & $\begin{array}{l}\text { All } \\
n=185(\%)\end{array}$ & $\begin{array}{l}\text { PI } \\
n=47(\%)\end{array}$ & $\begin{array}{l}\text { PEI } \\
n=138(\%)\end{array}$ & $p^{*}$ \\
\hline Surgical site infection & $42(22.7)$ & $12(25.5)$ & $30(21.7)$ & 0.687 \\
\hline Partial skin necrosis & $41(22.2)$ & $10(21.3)$ & $31(22.5)$ & 1.000 \\
\hline Blood loss requiring transfusion & $19(10.3)$ & $3(6.4)$ & $16(11.6)$ & 0.411 \\
\hline Hematoma requiring reoperation & $10(5.4)$ & $2(4.3)$ & $8(5.8)$ & 1.000 \\
\hline Non-breast related complicationsb & $6(3.2)$ & 0 & $6(4.3)$ & 0.340 \\
\hline Seroma requiring evacuation & $5(2.7)$ & $1(2.1)$ & $4(2.9)$ & 1.000 \\
\hline Wound dehiscence & $2(1.1)$ & $1(2.1)$ & $1(0.7)$ & 0.445 \\
\hline Total no. of womena & $88(47.6)$ & $19(40.4)$ & $69(50.0)$ & 0.311 \\
\hline
\end{tabular}

$B R R M$ bilateral risk-reducing mastectomy, IBR immediate breast reconstruction, $P I$ permanent fixed volume implant, $P E I$ permanent expander implant

*The difference between PI and PEI

${ }^{a}$ Some women had more than one complication

${ }^{b}$ Pneumothorax (2), pneumonia (1), urinary tract infection requiring hospitalization (1), fall accident with suspected nerve injury (1), subluxation of shoulder [1]

occurred in 41 patients (22.2\%). In three cases, the necrosis was more severe and required surgery (one fasciocutaneous flap, one implant removal due to necrosis and infection and one local excision). Nineteen (10.3\%) participants required red blood cell transfusion. The overall complication rate was not significantly different between the PEI $(50.0 \%)$ and the PI $(40.4 \%)$ groups $(p=0.311)$.

In a multivariate analysis including age (continuous), BMI ( $<25, \geq 25$ ), smoking (yes, no), implant type (PEI, $\mathrm{PI}$ ), incision technique (the oval excision and the periareolar incision grouped together versus the modified Wise pattern incision), operation time (continuous) and specimen weight $<350 \mathrm{~g}, \geq 350 \mathrm{~g}$ ), the only factor associated with an increased risk of an early complication was BMI of $\geq 25$ (OR 7.1, 95\% CI 2.44-20.4).

\section{Reoperations within 2 years}

In total, $60 \%$ of the women underwent at least one unanticipated surgical procedure within 2 years of BRRM. The cumulative reoperation-free 2-year survival was significantly higher in the PI than in the PEI group ( $81 \%$ vs. $26 \%$, $p<0.001$ ) (Fig. 5). When only surgeries involving the implant pocket were included, the difference decreased but remained statistically significant ( $89 \%$ vs. $43 \%, p<0.001$ ) (Fig. 6). The results described in Figs. 5 and 6 did not change when adjusting for age, BMI, smoking, incision type, operation time and specimen weight in a multivariate analysis. The relative risk of any unanticipated reoperation within 2 years for PI versus PEI was $0.33(95 \%$ CI $0.15-0.73$ ). When only reoperations involving the implant pocket were included, the relative risk for PI versus PEI was 0.21 (95\% CI 0.07-0.58). In these analyses, BMI $\geq 25$ (RR 2.15; 95\% CI 1.26-3.67) and smoking (RR 1.92; 95\%

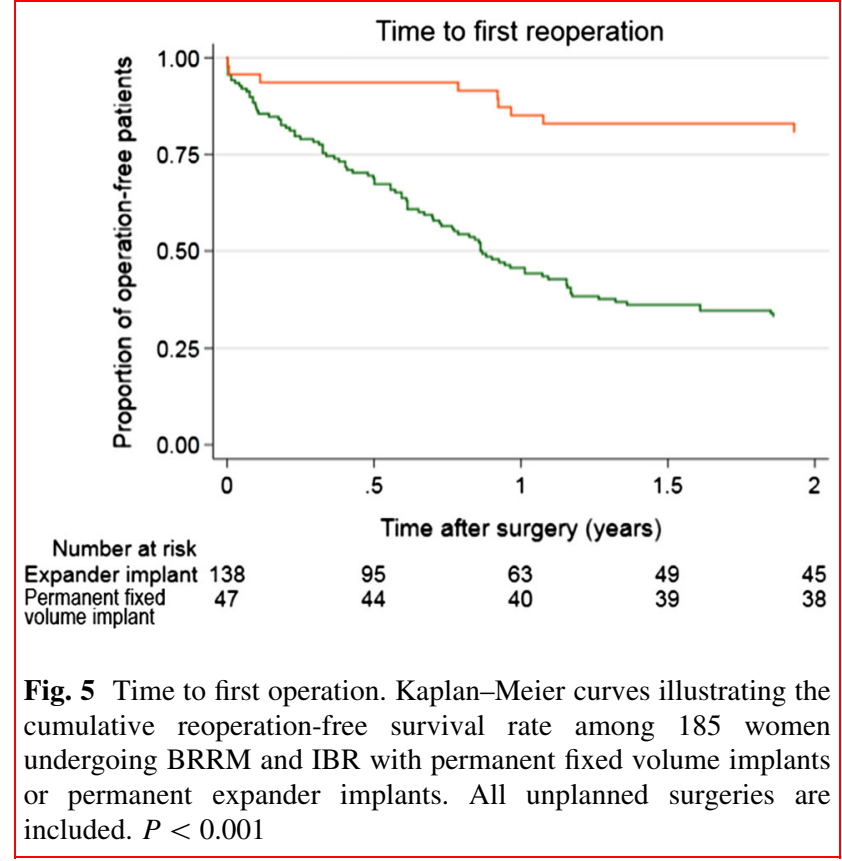

CI 1.14-3.23) were factors associated with an increased risk of any unanticipated reoperation, and BMI $\geq 25$ (RR 1.96; 95\% CI 1.05-3.65) was associated with an increased risk of reoperation involving the implant pocket.

\section{Implant loss}

In 19 patients $(10 \%)$, one $(n=16)$ or both $(n=3)$ implants had to be removed at some point during the full reconstruction period (modified Wise pattern $n=7$, periareolar incision $n=7$, oval excision $n=5$ ). All but one were PEI reconstructions. Ten of the patients had the implant/s 


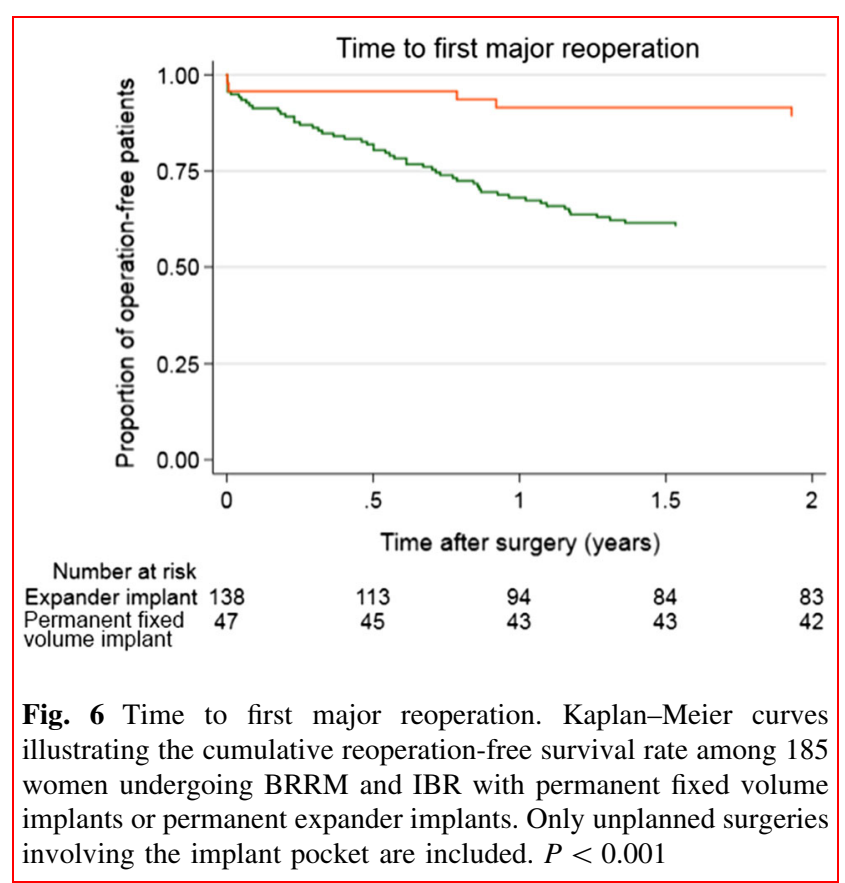

removed due to infection after the primary reconstruction. Other causes for implant removal were infection after corrective surgeries, late infection of unknown cause or implant rupture. At the end of follow-up, all but four had received a new implant.

\section{Healthcare utilization}

The mean hospital stay after BRRM and IBR was 5.0 days (range $3-11$ days) and was slightly longer in the PEI than in the PI group (5.1 vs. 4.5 days, $p=0.007$ ). There was a significant difference in median reconstruction time between PEI and PI (12.4 vs 1.0 months, $p<0.001$ ). Women undergoing mastectomy with removal of the NAC (and regrafting of the nipple tip) in combination with PEI had a median reconstruction time of 14.3 months (range 5.4-173.7 months). Women undergoing mastectomy with preservation of the NAC in combination with PI had a median reconstruction time of 1.0 months (range 0.9-18.9). Including the primary surgery, each woman underwent a mean number of 2.3 operations (range 1-7) and made 10.8 outpatient visits to see either a surgeon or a nurse (range 0-39) before the reconstruction was considered complete. Patients with PI required significantly fewer operations (1.3 vs. 2.6, $p<0.001$ ) and outpatient visits (3.9 vs. 13.3, $p<0.001$ ). After completed reconstruction, there was a continuing healthcare need due to outpatient visits, corrective surgeries and late complications (mean no. of operations 0.5 , mean no. of visits 3.3).

\section{Clinical follow-up}

A total of 175 women $(94.6 \%)$ had completed the breast reconstruction at the end of follow-up. Nine women were awaiting further procedures (i.e., expansions, revision surgeries, tattooing) and one woman had corrective surgery in a private institution.

One woman developed disseminated adenocarcinoma during follow-up, as previously reported [7]. Six women were diagnosed with different malignancies-one lung cancer, one rectal cancer, one pancreatic cancer, two ovarian cancers and one recurrence of a previously treated ovarian cancer. Six of these seven women are deceased. The remaining 178 women were alive by December 2016.

Regarding patient satisfaction and reported symptoms, which have been published previously, generally patients report high satisfaction with the procedure but long-term bodily problems have been reported $[8,9,16,17]$.

\section{Discussion}

This study supports that BRRM and IBR with implants are oncologically good options in previously healthy women with increased risk of breast cancer. A BMI $\geq 25$ is a risk factor for complications and reoperations regardless of implant type used. The study also shows that when PIs are used, the reconstruction time is short and the number of corrective surgeries is significantly fewer within a 2-year period than when the patient receives PEI. During the 18-year observation time, increasing rates of skin-sparing mastectomies were performed. In the present study, only one woman developed and succumbed from a disseminated adenocarcinoma after BRRM and PSOE despite having undergone a non-skin-sparing mastectomy. This is well in accordance with reported studies showing a risk reduction of $95 \%$ [3, 13]. There was a clear trend over time toward using PI when feasible, especially after the introduction of textured anatomically shaped implants. When performing the statistical analyses in the study, a breast volume of $350 \mathrm{ml}$ was randomly set and based on clinical experience. As many patients in the study were slim and had relatively small breasts, they could have been candidates for PI had these been available in an anatomical shape in the early phase of the study. This would also have affected the resource consumption as the reconstruction could have been completed after the primary operation. Expander implants are still predominantly used for women with larger breasts and higher BMI as also shown in this study. During the study period, the prevailing reconstruction method was that of using PEI and removal of the filling ports thus completed the reconstruction. However, these patients often underwent several revision surgeries. Current 
practice is to use a two-stage expander implant in order to prevent further revisions. Due to the increased complication rates in overweight and obese women, immediate reconstruction is usually not recommended until after weight loss. The short-term complication rate was equal between the two implant types. Single dose of antibiotics as prophylaxis has been a routine since IBR was introduced. There is no evidence to support other administration form or prolonged use. An unpublished randomized trial in our unit is evaluating single versus multiple doses in the prophylactic setting. Autologous reconstructions were practically never performed during the study period. Currently, women who are suitable and demand autologous reconstruction double DIEP flaps are offered. In line with other studies [18], implant loss rate was more prevalent among obese women. Necrosis was more prevalent after NAC preservation with areolae incisions but was not associated with the type of implant used. During the study period, antibiotic prophylaxis was administered to all patients as a single infusion. There is currently no evidence to suggest any other mode of administration. More than half of the operated women were BRCA1/2 mutation carriers, and the rest of the women had a substantially increased hereditary risk; thus, the surgical indication was strict. The preoperative planning and workup followed a structured concept including preoperative imaging where MRI became routine from 2006. No case of anaplastic large cell lymphoma has been reported in this series, and currently, no routines exist to remove textured implants.

\section{Methodological considerations}

This study represents a large single 18-year institutional experience of BRRM and IBR. Only asymptomatic highrisk women were included. Few surgeons were involved which may have influenced the outcome, a strength as well as a limitation of the study. During the first 14 years of the study period, PEI dominated while PI became more common the last 4 years. This may have introduced bias because of temporal changes in surgical practice, patient selection etcetera that we could not fully control for. We have, however, no reason to believe that such bias would be strong enough to explain the substantially lower rate of reoperations and resource consumption we observed in women undergoing reconstruction with PI versus PEI. The follow-up time was shorter in the PI group thus not fully justifying a comparison. However, 175 of the 185 women completed their reconstruction and most of the resource consumption metrics presented are measured up until the time of reconstruction completion. According to the hazard ratio implant survival analyses, it is definitely resource saving using PI when applicable.

\section{Conclusion}

Implant-based reconstruction remains a valid option after BRRM in high-risk women. Whenever possible (low BMI and small breast volume without severe ptosis), permanent implants can be safely recommended and resource saving.

Funding The study was supported by grants from Paola BrunettiJacovone bequest, Ragnar and Jacob Söderberg Research Fund and Stockholm County Research Fund ALF.

Data availability statement The dataset used to support the findings of this study has been deposited in the Department of Molecular Medicine and Surgery Karolinska Institutet repository.

\section{Compliance with ethical standards}

Informed consent Additional informed consent was obtained from all participants for whom identifying information is included in the article.

Open Access This article is distributed under the terms of the Creative Commons Attribution 4.0 International License (http://crea tivecommons.org/licenses/by/4.0/), which permits unrestricted use, distribution, and reproduction in any medium, provided you give appropriate credit to the original author(s) and the source, provide a link to the Creative Commons license, and indicate if changes were made.

\section{References}

1. Carbine NE, Lostumbo L, Wallace J, Ko H (2018) Risk-reducing mastectomy for the prevention of primary breast cancer. Cochrane Database Syst Rev. 4:002748

2. Hartmann LC, Sellers TA, Schaid DJ, Frank TS, Soderberg CL, Sitta DL et al (2001) Efficacy of bilateral prophylactic mastectomy in BRCA1 and BRCA2 gene mutation carriers. J Natl Cancer Inst. 93(21):1633-1637

3. Rebbeck TR, Friebel T, Lynch HT, Neuhausen SL, van't Veer L, Garber JE et al (2004) Bilateral prophylactic mastectomy reduces breast cancer risk in BRCA1 and BRCA2 mutation carriers: the PROSE Study Group. J Clin Oncol 22(6):1055-1062

4. Brandberg Y, Sandelin K, Erikson S, Jurell G, Liljegren A, Lindblom A et al (2008) Psychological reactions, quality of life, and body image after bilateral prophylactic mastectomy in women at high risk for breast cancer: a prospective 1-year followup study. J Clin Oncol. 26(24):3943-3949

5. den Heijer M, Seynaeve C, Timman R, Duivenvoorden HJ, Vanheusden K, Tilanus-Linthorst M et al (2012) Body image and psychological distress after prophylactic mastectomy and breast reconstruction in genetically predisposed women: a prospective long-term follow-up study. Eur J Cancer. 48(9):1263-1268

6. Skytte AB, Gerdes AM, Andersen MK, Sunde L, BrondumNielsen K, Waldstrom $M$ et al (2010) Risk-reducing mastectomy and salpingo-oophorectomy in unaffected BRCA mutation carriers: uptake and timing. Clin Genet. 77(4):342-349

7. Arver B, Isaksson K, Atterhem H, Baan A, Bergkvist L, Brandberg Y et al (2011) Bilateral prophylactic mastectomy in Swedish 
women at high risk of breast cancer: a national survey. Ann Surg. 253(6): 1147-1154

8. Gahm J, Hansson P, Brandberg Y, Wickman M (2013) Breast sensibility after bilateral risk-reducing mastectomy and immediate breast reconstruction: a prospective study. J Plast Reconstr Aesthet Surg. 66(11):1521-1527

9. Wasteson E, Sandelin K, Brandberg Y, Wickman M, Arver B (2011) High satisfaction rate ten years after bilateral prophylactic mastectomy - a longitudinal study. Eur J Cancer Care (Engl) 20(4):508-513

10. Wickman M, Sandelin K, Arver B (2003) Technical aspects and outcome after prophylactic mastectomy and immediate breast reconstruction in 30 consecutive high-risk patients. Plast Reconstr Surg. 111(3):1069-1077

11. Gahm J, Jurell G, Edsander-Nord A, Wickman M (2010) Patient satisfaction with aesthetic outcome after bilateral prophylactic mastectomy and immediate reconstruction with implants. J Plast Reconstr Aesthet Surg. 63(2):332-338

12. Jakub JW, Peled AW, Gray RJ, Greenup RA, Kiluk JV, Sacchini V et al (2018) Oncologic safety of prophylactic nipple-sparing mastectomy in a population with BRCA mutations: a multi-institutional study. JAMA Surg. 153(2):123-129

13. Kaas R, Verhoef S, Wesseling J, Rookus MA, Oldenburg HS, Peeters MJ et al (2010) Prophylactic mastectomy in BRCA1 and BRCA2 mutation carriers: very low risk for subsequent breast cancer. Ann Surg. 251(3):488-492
14. Manning AT, Sacchini VS (2016) Conservative mastectomies for breast cancer and risk-reducing surgery: the Memorial Sloan Kettering Cancer Center experience. Gland Surg. 5(1):55-62

15. Brandberg Y, Arver B, Lindblom A, Sandelin K, Wickman M, Hall P (2004) Preoperative psychological reactions and quality of life among women with an increased risk of breast cancer who are considering a prophylactic mastectomy. Eur $\mathrm{J}$ Cancer. 40(3):365-374

16. Bai L, Arver B, Johansson H, Sandelin K, Wickman M, Brandberg Y (2019) Body image problems in women with and without breast cancer 6-20 years after bilateral risk-reducing surgery-a prospective follow-up study. Breast 44:120-127

17. Gahm J, Edsander-Nord A, Jurell G, Wickman M (2010) No differences in aesthetic outcome or patient satisfaction between anatomically shaped and round expandable implants in bilateral breast reconstructions: a randomized study. Plast Reconstr Surg. 126(5):1419-1427

18. McCarthy CM, Mehrara BJ, Riedel E, Davidge K, Hinson A, Disa JJ et al (2008) Predicting complications following expander/ implant breast reconstruction: an outcomes analysis based on preoperative clinical risk. Plast Reconstr Surg. 121(6):1886-1892

Publisher's Note Springer Nature remains neutral with regard to jurisdictional claims in published maps and institutional affiliations. 